\title{
Parametric linear quadratic control and random delays
}

\author{
P.M.Mäkilä and T.Norlander
}

\begin{abstract}
The authors derive the design equations for parametric linear quadratic control of continuous-time systems with random delays. This problem motivates a more general setup that is utilised for the development of efficient numerical algorithms. Systems with random delays are important in control problems over communication networks. The generalised setup allows robustness issues against modelling errors and statistically varying disturbances to be addressed. The results are illustrated with examples.
\end{abstract}

\section{Introduction}

Modern optimal analytical control theory provides a beautiful set of solutions to a number of important control problems [1-3]. However, there are a number of important design problems that appear to be out of reach by purely analytical methods. One of the famous unsolved problems is the optimal instantaneous output feedback problem [2, 3] (unsolved in an analytical sense). This problem is a special case of parametric linear quadratic control [4]. The steady increase in computing power will make it likely that systematic nonanalytical approaches gain more prominence in the control and systems field in the future.

The parametric linear quadratic (PLQ) (and $\mathrm{H}_{2}$ ) control approach offers a versatile set of tools to solve many realistic controller design problems; see, for example, [46] and the references therein. This is in fact an old field; see, for example, [2-4, 7] for some historical remarks. Similar techniques have also been used in parametric $H_{\infty}$ control. In [8] a re-examination of infinite horizon LQ control is given with generalised nonprobabilistic disturbance [9] and loss function descriptions.

There are many possible numerical approaches for solving parametric LQ control problems [4]. Unfortunately, parametric LQ problems are not generically convex problems. Hence it is well justified to approach the solution of parametric LQ problems with methods that in the case of a nice convex PLQ problem instance are guaranteed to find the solution. Many approaches have in fact been used, such as homotopy methods, variable metric gradient methods, the Newton method, and special purpose methods.

\section{(C) IEE, 2000}

IEE Proceedings online no. 20000762

DOI: $10.1049 /$ ip-cta: 20000762

Paper first received 8th February and in revised form 26th June 2000

P.M. Mäkilä is with the Automation and Control Institute, Tampere University of Technology PO Box 692, FIN-33101 Tampere, Finland E-mail: pmakila@ad.tut.fi

T. Norlander is with the Control Engineering Group, Luleå University of Technology, S-97187 Luleå, Sweden, and Volvo Aero Corporation, S-461 81 Trollhättan, Sweden

E-mail: vac.tono@memo.volvo.se
In this work we illustrate how to derive in a systematic manner very simple and efficient algorithms to solve certain difficult design problems. We start by considering the optimal instantaneous output feedback problem for systems with time-varying (or random) delays. We then show that this problem formulation motivates a more general setup which could be applied to consider robustness issues like modelling errors and statistically varying disturbances. The reason for considering time-varying delays is that they are a growing problem in industrial applications and that they probably will be more prominent in industrial applications in the future. These delays, which are often of a stochastic nature, i.e. randomly varying, are likely to occur when the control system is closed with a communication network. The assumption in standard controller design methods is that the system is timeinvariant. However, the presence of time-varying delays in the feedback loop may result in undesired closed-loop behaviour.

Time-varying (or random) delays in communication networks are evident in very fast processes, such as flight control systems in advanced aircraft [10] and active suspension in automobiles [11]. However, it has been shown in [12] that, as the number of network users increases, the augmented traffic causes a larger data latency, resulting in poorer performance even for relatively slow processes which are attached to the network. The phenomenon of time-varying delays is also likely to occur when the controller is implemented in a time-shared computer or when the computation time varies for each new value of the control signal that is computed, for example in fuzzy controllers or controllers based on artificial intelligence (AI); see [13].

It has been shown several times that time-varying delays can degrade the performance of the closed-loop system so that instability occurs $[11,14,15]$. It is therefore surprising that there is relatively little work which has been published on this topic. Because of the more frequent use of various networks and computing media in digital control systems, it is becoming more important to account for the random delays generated by the transmission of signals through such media in the controller design process. For more references on this topic see $[16,17]$.

We are here going to use certain special purpose numerical methods for PLQ problems partly because of our long 
experience with such methods. These methods rely quite heavily on the special structure of PLQ problems and involve therefore a certain amount of analytic work, yet are very simple to implement in algorithmic form. One advantage of these methods is that they provide more information about the design problem than an analytical solution alone as they generate a sequence of controllers and their associated performances. Typically problems in the numerical solution indicate lack of robustness and related risks in problem formulation.

A parametric LQ control problem for linear continuoustime systems with random delays is studied in Section 2. It is shown that the particular problem studied here is a special case of a more general parametric LQ problem for linear systems with stochastic parameters. Descent Anderson-Moore type algorithms are described in detail for this latter class of problems in Section 3. Numerical examples are presented in Section 4. These illustrate that the considered general setup allows that robustness issues against not only random delays, but also against modelling errors; unmodelled dynamics and disturbances with timevarying statistical properties can be addressed within the considered setup.

\section{Parametric LQ control with random delays}

Consider the linear finite-dimensional single-input system

$$
\begin{aligned}
d x(t) & =A x(t) d t+B u(t) d t+d v(t) \\
y(t) & =C x(t)+e(t)
\end{aligned}
$$

where $x$ is the state, $u$ is the input, $y$ is the output, and $v$ and $e$ are disturbance (noise) terms. Here $v$ is a Wiener process with incremental covariance matrix $R_{v}$. Finally, $e$ is a white noise process with covariance matrix $R_{e}$ and independent of $v$. (Note that the assumptions on $e$ imply that the sample paths of $e$ are not continuous functions of time). Eqn. 1 is a system of linear stochastic differential equations [1].

It is assumed that the sensors sample $y$ each $h$ time unit in a syncronised manner and send the sampled $y$ values via a common medium to a controller node that computes the next control signal $(u)$ value, which is then sent to an actuator. Let $\tau_{k}^{s c} \geq 0$ and $\tau_{k}^{c a} \geq 0$ denote the time delays between the sensor and the controller node and between the controller node and the actuator, respectively, on the sampling interval $[k h,(k+1) h)$. Here $h$ denotes the length of the sampling interval. It is assumed that

$$
\tau_{k}^{s c}+\tau_{k}^{c a}<h
$$

The sampled system is then given in a standard manner as

$$
x((k+1) h)=\Phi x(k h)+\Gamma_{0} u(k h)+\Gamma_{1} u((k-1) h)+w(k h)
$$

where $\Phi=\exp A h$ and

$$
\begin{aligned}
& \Gamma_{0}\left(\tau_{k}^{s c}, \tau_{k}^{c a}\right)=\int_{0}^{h-\tau_{k}^{s c}-\tau_{k}^{c a}} e^{A s} d s B \\
& \Gamma_{1}\left(\tau_{k}^{s c}, \tau_{k}^{c a}\right)=\int_{h-\tau_{k}^{s c}-\tau_{k}^{c a}}^{h} e^{A s} d s B
\end{aligned}
$$

Furthermore, $\{w(k h)\}$ is a white noise process with covariance matrix

$$
R_{w}=\mathrm{E}\left\{w(k h) w(k h)^{\prime}\right\}=\int_{0}^{h} e^{A(h-s)} R_{v} e^{A^{\prime}(h-s)} d s
$$

Let $\left\{\tau_{k}^{s c}\right\}$ and $\left\{\tau_{k}^{c a}\right\}$ be independent white noise sequences such that eqn. 3 is satisfied. Note that if the condition in eqn. 3 does not hold then the sampled system eqn. 4 contains older inputs than $u((k-1) h)$. We leave the analysis of this more general case to future work.

Let us assume that the control law is given by

$$
u(k h)=F y(k h)
$$

We shall be interested in the quadratic loss function

$$
\mathrm{E}\left[x((k+1) h)^{\prime} Q x((k+1) h)+u(k h)^{\prime} R u(k h)\right]
$$

where $Q$ and $R$ are (symmetric) positive semidefinite weighting matrices and the expectation operation $E$ is taken over all stochastic variables present in the continuous system, eqns. 1 and 2, and the sampled system, eqn. 4 .

Furthermore, we shall be interested in the limiting value of eqn. 9 when $k \rightarrow \infty$. This limit exists when the second moment matrix of $x(\mathrm{kh})$ approaches a constant (finite) matrix for $k \rightarrow \infty$. A necessary condition for this is that $A+\Gamma_{0}(0,0) F C$ is a stable matrix. Hence it is assumed that the associated delay free discretised system is stabilisable by instantaneous output feedback. To study the existence of the limiting value of eqn. 9 let us start by deriving a second moment equivalent system to eqn. 4.

Theorem 1. The system eqn. 4 has the same second moments as the system

$$
\begin{aligned}
& {\left[\begin{array}{c}
x((k+1) h) \\
u(k h)
\end{array}\right]} \\
& =\left[\begin{array}{cc}
\Phi & \Gamma_{1, a}+\sum_{i=1}^{n} \eta_{i}(k)\left(-\Gamma_{i}^{(0)}\right) \\
0 & 0
\end{array}\right]\left[\begin{array}{c}
x(k h) \\
u((k-1) h)
\end{array}\right] \\
& \quad+\left[\begin{array}{c}
\Gamma_{0, a}+\sum_{i=1}^{n} \eta_{i}(k) \Gamma_{i}^{(0)} \\
1
\end{array}\right] u(k h)+\left[\begin{array}{c}
w(k h) \\
0
\end{array}\right]
\end{aligned}
$$

where

$$
\begin{aligned}
& \Gamma_{0, a}=\mathrm{E} \Gamma_{0}\left(\tau_{k}^{s c}, \tau_{k}^{c a}\right) \\
& \Gamma_{1, a}=\mathrm{E} \Gamma_{1}\left(\tau_{k}^{s c}, \tau_{k}^{c a}\right)=\int_{0}^{h} e^{A s} d s B-\Gamma_{0, a}
\end{aligned}
$$

Furthermore, $\left\{\eta_{i}\right\}_{i=1}^{n}$ are independent white noise processes with zero means and variances $\left\{\sigma_{i}^{2}\right\}$. Here $n=\operatorname{dim} x$. The variances $\left\{\sigma_{i}^{2}\right\}$ are the eigenvalues (arranged in decreasing order) of the (symmetric) positive semidefinite matrix

$$
H=\mathrm{E}\left[\Gamma_{0}\left(\tau_{k}^{s c}, \tau_{k}^{c a}\right) \Gamma_{0}\left(\tau_{k}^{s c}, \tau_{k}^{c a}\right)^{\prime}\right]-\Gamma_{0, a} \Gamma_{0, a}^{\prime}
$$

and the vectors $\left\{\Gamma_{i}^{(0)}\right\}$ are the orthonormal eigenvectors of $H$. That is, $H \Gamma_{i}^{(0)}=\sigma_{i}^{2} \Gamma_{i}^{(0)}$.

Proof: Simply evaluate $\mathrm{E}\left[x((k+1) h) x((k+1) h)^{\prime}\right]$ (i.e. the second moment matrix of $x((k+1) h))$ via both eqns. 4 and 10 and compare the results. The essential assumption for the two results to be the same is that the delays $\tau_{k}^{s c}$ and $\tau_{k}^{c a}$ are independent white noise processes (actually it would suffice to assume that they are uncorrelated). Recall also that the input $u$ is one-dimensional by assumption.

Note that eqn. 10 is a special case of a class of state space systems with stochastic parameters that has been been studied earlier; see, for example, $[5,18]$ and the references therein. Hence we can write up directly, using eqn 10, the expressions for the limiting value of $\mathrm{E}\left[x((k+1) h)^{\prime} Q x((k+1) h)+u(k h)^{\prime} R u(k h)\right] \quad$ when the system is controlled with eqn. 8 for a feedback gain 
matrix $F$ such that the limiting value exists, but before we do this let us evaluate the vectors $\Gamma_{0, a}, \Gamma_{1, a}$ and the matrix $H$ in an interesting special case.

Hence, let $\tau_{k}^{s c}$ and $\tau_{k}^{c a}$ be uniformly distributed in the interval $\left[0, \tau_{\text {max }}^{s c}\right]$ and in the interval $\left[0, \tau_{\text {max }}^{c a}\right]$, respectively. Let us furthermore assume for convenience that the continuous-time system matrix $A$ in eqn. 1 has an inverse. Then it is straightforward to evaluate

$$
\Gamma_{0, a}=A^{-1}\left[\frac{A^{-2} e^{A h}}{\tau_{\max }^{s c} \tau_{\max }^{c a}}\left(e^{-A \tau_{\max }^{s c}}-I\right)\left(e^{-A \tau_{\text {max }}^{c a}}-I\right)-I\right] B
$$

This gives $\Gamma_{1, a}$ as

$$
\Gamma_{1, a}=A^{-1}\left(e^{A h}-I\right) B-\Gamma_{0, a}
$$

To evaluate $H$ we need to evaluate $G=\mathrm{E}\left[\Gamma_{0}\left(\tau_{k}^{s c}, \tau_{k}^{c a}\right)\right.$ $\left.\Gamma_{0}\left(\tau_{k}^{s c}, \tau_{k}^{c a}\right)^{\prime}\right]$. Clearly

$$
G=\mathrm{E}\left[A^{-1}\left(e^{A\left(h-\tau_{k}^{s c}-\tau_{k}^{c i}\right)}-I\right) B B^{\prime}\left(e^{A^{\prime}\left(h-\tau_{k}^{s c}-\tau_{k}^{c a}\right)}-I\right)\left(A^{-1}\right)^{\prime}\right]
$$

The expectation $\mathrm{E}$ above can be integrated numerically as a double (matrix-valued) integral over the square $\left[0, \tau_{\max }^{s c}\right] \times\left[0, \tau_{\max }^{c a}\right]$. When $A^{-1}$ does not exist, it is possible to either evaluate all integrals purely numerically, or to perturb $A$ slightly to an invertible matrix $A+\Delta A$ and to proceed as above and to estimate the error caused by the perturbation.

\section{A more general setup}

As noted earlier the system eqn. 10, with its output equation and the control law, eqn. 8 , is a special case of

$$
\begin{aligned}
x(k+1)= & \left(A+\sum_{i=1}^{m} \eta_{i}(k) A_{i}\right) x(k) \\
& +\left(B+\sum_{i=1}^{m} \eta_{i}(k) B_{i}\right) u(k)+v(k) \\
y(k)= & C x(k)+e(k) \\
u(k)= & F y(k)
\end{aligned}
$$

where $v$ and $e$ are uncorrelated zero mean white noise processes with covariance matrices $R_{v}$ and $R_{e}$, respectively. Furthermore, $\left\{\eta_{i}\right\}_{i=1}^{m}$ are uncorrelated zero mean white noise processes with variances $\left\{\sigma_{i}^{2}\right\}_{i=1}^{m}$. (Here $\eta_{i}=\left\{\eta_{i}(k)\right\}_{k \geq 0}$.) Note that here the notation is the standard one for discrete time systems, but this should not cause any confusion with the notation in eqns. 1 and 10 . Note that here the input $u$ can be a vector.

Introduce the loss function

$$
J(F)=\lim _{k \rightarrow \infty} \mathrm{E}\left[x(k+1)^{\prime} Q x(k+1)+u(k)^{\prime} R u(k)\right]
$$

where $Q$ and $R$ are (symmetric) positive semidefinite weighting matrices. Assuming that $P=P(F)=\lim _{k \rightarrow \infty}$ $x(k) x(k)^{\prime}$ exists, the loss $J(F)$ can be written as

$$
J(F)=\operatorname{tr}\left(Q+C^{\prime} F^{\prime} R F C\right) P+\operatorname{tr} F^{\prime} R F R_{e}
$$

Furthermore, $P$ is given as a positive semidefinite solution to the generalised Lyapunov equation

$$
\begin{aligned}
P= & (A+B F C) P(A+B F C)^{\prime}+B F R_{e} F^{\prime} B^{\prime}+R_{v} \\
& +\sum_{i=1}^{m} \sigma_{i}^{2}\left[\left(A_{i}+B_{i} F C\right) P\left(A_{i}+B_{i} F C\right)^{\prime}+B_{i} F R_{e} F^{\prime} B_{i}^{\prime}\right]
\end{aligned}
$$

One method to solve this matrix equation is to write it as a system of linear equations (unfortunately even if it is utilised that $P$ is symmetric, the resulting system of equations has $\left(n^{2}+n\right) / 2$ unknowns, where $\left.n=\operatorname{dim} x\right)$, and solve this system with any standard method such as Gauss elimination. For more details see [19]. But as the number of unknowns grows as $n^{2}$ this approach is not very attractive for large $n$. Another approach is to solve eqn. 22 iteratively by defining the sequence

$$
\begin{aligned}
P_{0}= & B F_{e} F^{\prime} B^{\prime}+R_{v}+\sum_{i=1}^{m} \sigma_{i}^{2} B_{i} F R_{e} F^{\prime} B^{\prime} \\
P_{j+1}= & (A+B F C) P_{j+1}(A+B F C)^{\prime}+B F R_{e} F^{\prime} B^{\prime}+R_{v} \\
& +\sum_{i=1}^{m} \sigma_{i}^{2}\left[\left(A_{i}+B_{i} F C\right) P_{j}\left(A_{i}+B_{i} F C\right)^{\prime}+B_{i} F R_{e} F^{\prime} B_{i}^{\prime}\right], \\
& j \geq 0
\end{aligned}
$$

where $P_{j+1}$ is obtained by solving a standard Lyapunov equation with some efficient method (note that one can utilise repeatedly the same matrix transformation of $A+B F C$ to the Schur form, for example, as in the Bartels-Stewart method). $P_{j}$ converges to $P$ when $P$ exists.

Let $S M_{F}$ denote the set of feedback gains for which $A+B F C$ is a stable matrix and for which $P=P(F)$ exists as a (finite) positive semidefinite matrix. (The notation $S M$ comes from $S M_{F}$ being the set of feedback gains stabilising the system eqn. 17 in the sense of finite second moments.) This set is a subset of the feedback gains for which $A+B F C$ is a stable matrix. Consider now the problem of minimising $J(F)$ over $S M_{F}$. Denote

$$
J_{i n f}=\inf _{F \in S M_{F}} J(F)
$$

Our objective is not to describe conditions for the existence of a minimiser $F_{o p} \in S M_{F}$ such that $J\left(F_{o p}\right)=J_{i n f}$. It suffices to state that such a minimiser exists under mild conditions. Our interest here is to discuss how to find such a solution when $S M_{F}$ is nonempty.

It would be possible to try numerical global optimisation of $J(F)$, but this is in general a computationally demanding task. In practice a designer wishes to have a fast practical approach for computing trial designs. Hence in practice it is typical, in many engineering fields, to use efficient iterative methods which guarantee that the user finds at least a local minimiser to a minimisation problem. These methods are such that when $S M_{F}$ is a (bounded) convex set and $J(F)$ is a strictly convex function in $S M_{F}$, they are guaranteed to find the global minimising feedback gain matrix $F_{o p}$. Wide experience from parametric LQ (linear quadratic) problems indicates that these two convexity conditions hold quite often, but not always.

A VERY special feature of the optimisation problem, eqn. 24 , is that it is possible to utilise the structure of $J(F)$ to an unusually great degree in the numerical minimisation. We describe here a technique that reduces to efficient methods for solving ordinary linear quadratic (LQ) control problems (and hence for solving algebraic Riccati equations) when the problem eqn. 24 reduces to such simpler 
and well understood problems. The main idea is to study the loss increment for the loss function $J$.

The loss increment technique was used first by Halyo and Broussard in their 1981 paper [20] on the optimal instantaneous output feedback problem. This paper provided an improvement over the Anderson-Moore method [2] for solving the optimal instantaneous output feedback problem. The first paper to analyse a provably (globally) convergent modification of the Anderson-Moore method is probably [21]. It became subsequently clear that the loss increment technique provides a natural way to derive (and to prove the convergence of) many promising algorithms for various parametric LQ control problems [4].

Let $F \in S M_{F}$. Introduce the (symmetric) positive semidefinite matrix $S=S(F)$ as a solution to the generalised Lyapunov equation

$$
\begin{aligned}
S= & (A+B F C)^{\prime} S(A+B F C)+C^{\prime} F^{\prime} R F C+Q \\
& +\sum_{i=1}^{m} \sigma_{i}^{2}\left(A_{i}+B_{i} F C\right)^{\prime} S\left(A_{i}+B_{i} F C\right)
\end{aligned}
$$

Introduce also the notation

$$
\begin{aligned}
& \hat{P}(F)=C P(F) C^{\prime}+R_{v} \\
& \hat{S}(F)=B^{\prime} S(F) B+R
\end{aligned}
$$

The matrix function $S(F)$ can be used to derive an expression for the loss increment $\Delta J\left(F_{2}, F_{1}\right)=J\left(F_{2}\right)-J\left(F_{1}\right)$ for any $F_{1}, F_{2} \in S M_{F}$. This involves only straightforward (but lengthy) algebraic manipulations with the result

$$
\begin{aligned}
\Delta J\left(F_{2}, F_{1}\right)= & \operatorname{tr} \Delta F^{\prime}\left\{2\left[\hat{S}\left(F_{1}\right) F_{1} \hat{P}\left(F_{2}\right)+B^{\prime} S\left(F_{1}\right) A P\left(F_{2}\right) C^{\prime}\right]\right. \\
& +\sum_{i=1}^{m} 2 \sigma_{i}^{2}\left[B_{i}^{\prime} S\left(F_{1}\right) B_{i} F_{1} \hat{P}\left(F_{2}\right)\right. \\
& \left.\left.+B_{i}^{\prime} S\left(F_{1}\right) A_{i} P\left(F_{2}\right) C^{\prime}\right]\right\} \\
& +\operatorname{tr} \Delta F^{\prime} \hat{S}\left(F_{1}\right) \Delta F \hat{P}\left(F_{2}\right) \\
& +\sum_{i=1}^{m} \sigma_{i}^{2} \operatorname{tr} \Delta F^{\prime} B_{i}^{\prime} S\left(F_{1}\right) B_{i} \Delta F \hat{P}\left(F_{2}\right)
\end{aligned}
$$

where $\Delta F=F_{2}-F_{1}$. Therefore the gradient of the loss $J(F)$ at $F \in S M_{F}$ is given by

$$
\begin{aligned}
\frac{\partial J}{\partial F}= & 2\left[\hat{S}(F) F \hat{P}(F)+B^{\prime} S(F) A P(F) C^{\prime}\right] \\
& +\sum_{i=1}^{m} 2 \sigma_{i}^{2}\left[B_{i}^{\prime} S(F) B_{i} F \hat{P}(F)+B_{i}^{\prime} S(F) A_{i} P(F) C^{\prime}\right]
\end{aligned}
$$

The loss increment expression suggests several methods for finding a minimiser of $J(F)$ in $S M_{F}$. The simplest of these methods is a direct generalisation of the descent Anderson-Moore method using a simple step length rule as follows.

\section{Algorithm DAMI:}

1. Let $F_{0} \in S M_{F}$ be an initialising feedback matrix. Choose $\epsilon>0$ and an integer iterm $>0$. Compute $J\left(F_{0}\right)$ and

$$
\left(\frac{\partial J}{\partial F}\right)_{0}
$$

Put $k=1$

2. If

$$
\left\|\left(\frac{\partial J}{\partial F}\right)_{k}\right\|<\epsilon
$$

then stop with success flag up else go to step 3 .
3. Set $\alpha=1$. If $k \geq i$ iterm stop with nonconvergence flag up else continue to step 4 .

4. Compute

$$
D_{k}=-\frac{1}{2} \hat{S}\left(F_{k}\right)^{-1}\left(\frac{\partial J}{\partial F}\right)_{k} \hat{P}\left(F_{k}\right)^{-1}
$$

5. Set $F_{k+1}=F_{k}+\alpha D_{k}$ and compute $J\left(F_{k+1}\right)$.

6. If $F_{k+1} \in S M_{F}$ and $J\left(F_{k+1}\right)<J\left(F_{k}\right)$ then set $k \leftarrow k+1$ and go to step 2 else set $\alpha \leftarrow 0.5 \alpha$ and go to step 5 .

\section{End of Algorithm DAMI.}

The loss increment expression suggests the following modified step 4 in algorithm DAM1:

$4^{\prime}$. Compute

$$
D_{k}=-\frac{1}{2}\left(\hat{S}\left(F_{k}\right)+\sum_{i=1}^{m} \sigma_{i}^{2} B_{i}^{\prime} S\left(F_{k}\right) B_{i}\right)^{-1}\left(\frac{\partial J}{\partial F}\right)_{k} \hat{P}\left(F_{k}\right)^{-1}
$$

The modified algorithm will be called here DAM2.

Let us point out some simple robustification ideas that are worth including in an actual computer implementation of the above algorithms. Step $4\left(4^{\prime}\right)$ of the two algorithms contains a potentially fragile numerical operation, namely the inversion of two matrices. For example, with a zero initial matrix $F_{0}$ these inverses do not sometimes exist, but we can modify the matrices to be inverted in the computation of $D_{k}$ by arbitrary additions to them of positive semidefinite matrices of appropriate dimensions without destroying the descent property of the computed search direction! We have actually replaced in algorithm DAM1 step 4 by the robustified version

$$
\begin{gathered}
D_{k}=-\frac{1}{2}\left[\hat{S}\left(F_{k}\right)+\rho \operatorname{tr} \hat{S}\left(F_{k}\right) \times I\right]^{-1}\left(\frac{\partial J}{\partial F}\right)_{k} \\
{\left[\hat{P}\left(F_{k}\right)+\rho \operatorname{tr} \hat{P}\left(F_{k}\right) \times I\right]^{-1}}
\end{gathered}
$$

where $\rho>0$ is a small positive number and $I$ is a generic notation for identity matrices. An analogous refinement has been included in algorithm DAM2.

The algorithms DAM1 and DAM2 utilise a simple condition for choosing the step length parameter $0<\alpha \leq 1$. To prove global convergence of the algorithms to a stationary point (zero gradient), it is required to use a more complicated step length condition (Mäkilä and Toivonen [4]), such as the Goldstein condition. (This, as the descent condition $J\left(F_{k+1}\right)<J\left(F_{k}\right)$ used in the above compact algorithms, only guarantees convergence of the loss function values $J\left(F_{k}\right)$, but not of the gradient values $(\partial J / \partial F)_{k} \rightarrow 0$, when $k \rightarrow \infty$. In practice these algorithms often work well.)

\section{Examples}

A number of examples are included here to illustrate the discussed concepts and algorithms. We have used MATLAB version 5.2 in our computations. The dlyap function of the Control Systems Toolbox has been employed to solve standard Lyapunov equations. We have used the positive definiteness enforcing parameter value $\rho=0.00001$ in the DAM algorithms in all our computations below.

IEE Proc.-Control Theory Appl., Vol. 147, No. 6, November 2000 


\subsection{Robustified minimum variance control example}

Consider minimum variance control of the ARMAX system [1, pp. 184-187],

$$
y(t+1)+a_{1} y(t)=u(t)+b_{1} u(t-1)+e(t+1)+c_{1} e(t)
$$

where $a_{1}=-0.7, b_{1}=0.99$ and $c_{1}=0.95$. Here $\{e(t)\}$ is a white noise sequence with variance $\sigma_{e}^{2}=1$. The controller minimising $\mathrm{E} y(t)^{2}$ is given by

$$
\begin{aligned}
u(t) & =\left(a_{1}-c_{1}\right) y(t)-b_{1} u(t-1) \\
& =-1.65 y(t)-0.99 u(t-1)
\end{aligned}
$$

This controller achieves $\mathrm{Ey}(t)^{2}=\sigma_{e}^{2}=1$. Unfortunately, the obtained closed-loop system performance is very sensitive to small errors and/or perturbations in the model eqn. 33 . In [1, pp. 185-187] the suboptimal controller

$$
u(t)=-0.684 y(t)-0.966 u(t-1)
$$

is obtained to reduce the sensitivity of the designed system against variations in the $b_{1}$, parameter. This controller gives the output variance $\mathrm{E} y(t)^{2}=1.93 \sigma_{e}^{2}=1.93$.

Let us illustrate how the methods discussed earlier in this paper can be used here. Thus let us assume that the system model does not exactly represent the true system but that there is some unmodelled dynamics. Specifically, let us design a minimum variance controller for the system

$$
\begin{aligned}
y(t+1)+a_{1} y(t)= & u(t)+b_{1} u(t-1) \\
& +b_{2}(t) u(t-2)+e(t+1)+c_{1} e(t)
\end{aligned}
$$

where $\left\{b_{2}(t)\right\}$ is a white noise sequence with variance $\sigma_{b}^{2}$ and independent of $\{e(t)\}$. Put $\sigma_{e}^{2}=1$ and $\sigma_{b}^{2}=0.01$. Defining the state vector $x(t)=[y(t), u(t-1), u(t-2)$, $e(t)]^{\prime}$, we can write eqn. 36 as

$$
\begin{aligned}
x(t+1)= & {\left[\begin{array}{cccc}
-a_{1} & b_{1} & b_{2}(t) & c_{1} \\
0 & 0 & 0 & 0 \\
0 & 1 & 0 & 0 \\
0 & 0 & 0 & 0
\end{array}\right] x(t) } \\
& +\left[\begin{array}{l}
1 \\
1 \\
0 \\
0
\end{array}\right] u(t)+\left[\begin{array}{l}
1 \\
0 \\
0 \\
1
\end{array}\right] e(t)
\end{aligned}
$$

Introducing the vector $z(t)=[y(t), u(t-1)]^{\prime}$ we see that we can compute the parametric minimum variance controller of the form $u(t)=F z(t)$ using the techniques developed in the previous section (note that $z$ replaces the notation $y$ in that section) by putting there $Q_{11}=1$ and all other elements in the $4 \times 4$ matrix $Q$ equal to zero, $R=0$, $R_{e}=0(2 \times 2$ zero matrix $)$,

$$
C=\left[\begin{array}{llll}
1 & 0 & 0 & 0 \\
0 & 1 & 0 & 0
\end{array}\right], R_{v}=\left[\begin{array}{llll}
1 & 0 & 0 & 1 \\
0 & 0 & 0 & 0 \\
0 & 0 & 0 & 0 \\
1 & 0 & 0 & 1
\end{array}\right] \sigma_{e}^{2}
$$

Finally, put $\left(A_{1}\right)_{13}=1$ and all other elements in the $4 \times 4$ matrix $A_{1}$ equal to zero and $B_{1}$ equal to a zero vector. Set $\sigma_{1}^{2}=\sigma_{b}^{2}$.
Note that the algorithms DAM1 and DAM2 are identical in this example! We initialised the algorithm DAM1 using $F_{0}=\left[\begin{array}{ll}0 & 0\end{array}\right]$ and $\epsilon=0.00005$. The algorithm produced the result

$$
F_{o p}=[-1.2885-0.9632]
$$

with $J\left(F_{o p}\right)=1.3105$. The number of loss function gradient evaluations ( $=$ number of iteration steps) was 62 and the average number of loss function evaluations per iteration step was 3.4839 (which means that the total number of loss function evaluations was 216). The obtained controller

$$
u(t)=-1.2885 y(t)-0.9632 u(t-1)
$$

gives for the nominal model eqn. 33 the output variance value $\mathrm{E} y(t)^{2}=1.2392$. The closed-loop system associated with eqn. 33 and this controller remains stable for $0.952 \leq b_{1} \leq 1.299$. The minimum variance controller, eqn. 34 , remains stabilising for the system eqn. 33 only for $0.9897 \leq b_{1} \leq 1.0260$ ! The controller, eqn. 35 , stabilises eqn. 33 for $0.916 \leq b_{1} \leq 2.450$.

To illustrate the performance of the DAM algorithms let us repeat the above computations but now with $R=0.5$. The DAM1 (and the DAM2) algorithm gives the result

$$
u(t)=-0.8785 y(t)-0.9484 u(t-1)
$$

and the associated optimal loss value is 2.0672 . The number of iteration steps was 104 and the average number of loss function evaluations per iteration step was 1.4808 . This indicates that the loss function was made more convex, and hence easier to optimise, by the inclusion of the nonzero input weight $R=0.5$. The computed controller gives the output variance $\mathrm{E} y(t)^{2}=1.6271$ for the nominal model, eqn. 33, with $b_{1}=0.99$ and stabilises the system eqn. 33 for $0.901 \leq b_{1} \leq 1.894$.

How does a P-controller perform for this example? For this purpose we just put $u(t)=F y(t)$ and redefine $C$ and $R_{e}$ accordingly. We are again interested in minimising the output variance for eqn. 36. Set $R=0, \epsilon=0.000001$ and $F_{0}=0$. Now the DAM1 (and DAM2) algorithm gives that

$$
u(t)=-0.4274 y(t)
$$

and the associated optimal loss value is 2.8725 . The number of iteration steps was 4 and the average number of loss evaluations per iteration step was 1 ! Hence we see that by changing the controller structure we have made the loss function much nicer and easier to minimise. This means that the computed controller probably has good robustness margins. Indeed, it stabilises eqn. 33 for $-1.701 \leq b_{1} \leq 2.339$. However, it gives the output variance $\mathrm{E} y(t)^{2}=2.8659$ for the nominal model eqn. 33 with $b_{1}=0.99$. This variance value provides a considerable reduction of the open loop output variance, which is 6.3382 .

One of the main advantages of the parametric LQ approach is that one can almost freely try out various controller structures. Typically, difficulties in the optimisation process for the DAM algorithms indicate either a bad choice of weighting matrices or possible robustness risks with the chosen controller structure or both. 


\subsection{MIMO example}

This example is from $[22,23]$, where an application on digital control of a dry process rotary cement kiln is described. The identified model was

$$
\begin{aligned}
y(t+1) & =\left[\begin{array}{cc}
0.914 & 0.0800 \\
-0.126 & 0.917
\end{array}\right] y(t) \\
& +\left[\begin{array}{cc}
2.091 & -0.0744 \\
-0.211 & -0.0156
\end{array}\right] u(t) \\
& +e(t+1)+\left[\begin{array}{ll}
0 & 0 \\
0 & c_{1}
\end{array}\right] e(t)
\end{aligned}
$$

where $c_{1}=0.715$ and $e$ is white noise with covariance matrix

$$
R_{e}=\left[\begin{array}{cc}
0.0644 & 0.000257 \\
0.000257 & 0.0214
\end{array}\right]
$$

The control criterion is from [22],

$$
J=\mathrm{E}\left[y(t+1)^{\prime} Q y(t+1)+u(t)^{\prime} R u(t)\right]
$$

where

$$
Q=\left[\begin{array}{ll}
1 & 0 \\
0 & 1
\end{array}\right], R=\left[\begin{array}{cc}
10.7 & 0 \\
0 & 0.0220
\end{array}\right]
$$

Now let $c_{1}=0.715+\Delta c_{1}(t)$, where $\Delta c_{1}(t)$ is a white noise process with variance $\sigma_{1}^{2}=0.02$. That is, we consider effects of variations in $c_{1}$, which may simulate, for example, the effects of varying raw material properties. Let the control law be

$$
u(t)=F y(t)
$$

By defining the state vector $x(t)=\left[y(t), e_{2}(t)\right]^{\prime}$ we can write the system eqn. 43 with the random $c_{1}$ parameter as a stochastic state space system with three states in the form described in the preceding section.

We computed the optimal feedback gain matrix $F$ using the DAM1 algorithm (in this example the DAM1 and DAM2 algorithms are again equivalent). The algorithm was initialised with a zero matrix $F_{0}=0, \epsilon=0.00001$. This resulted in

$$
F_{o p}=\left[\begin{array}{cc}
-0.1780 & 0.1256 \\
2.0855 & 1.9103
\end{array}\right]
$$

with $J\left(F_{o p}\right)=0.3637$. It took seven iteration steps and one loss function evaluation per iteration step to reach the result! The output and input variances with this controller (averaged also over the variability in $c_{1}$ ) are

$$
\begin{array}{ll}
\mathrm{E} y_{1}(t)^{2}=0.0935, & \mathrm{E} y_{2}(t)^{2}=0.1937 \\
\mathrm{E} u_{1}(t)^{2}=0.0039, & \mathrm{E} u_{2}(t)^{2}=1.5241
\end{array}
$$

Note that the LQG controller computed for the nominal system eqn. 43 with $c_{1}=0.715$ [22] has 12 coefficients but only marginally better nominal performance than the best proportional controller with four parameters only. A four parameter controller was implemented successfully [22, $23]$. The controller parameters $F_{o p}$ are actually close to the implemented controller, which shows that the implemented controller tolerates considerable variability in the disturbance parameter $c_{1}$ without significant loss of performance while keeping the input signal variations acceptable.

\subsection{Random delay system example}

Consider the open-loop unstable system

$$
\begin{gathered}
d x=\left[\begin{array}{cc}
0.21 & -0.03 \\
0 & -0.03
\end{array}\right] x+\left[\begin{array}{l}
1 \\
0
\end{array}\right] u+d v \\
y=\left[\begin{array}{ll}
1 & 0
\end{array}\right] x+e \\
u(k h)=F y(k h)
\end{gathered}
$$

where the incremental covariance matrix of $v$ is

$$
R_{v}=\left[\begin{array}{ll}
1 & 1 \\
1 & 1
\end{array}\right]
$$

The covariance matrix of $e$ is $R_{e}=0.01$. The weighting matrices are

$$
Q=\left[\begin{array}{ll}
1 & 0 \\
0 & 0
\end{array}\right]
$$

and $R=0.0001$. We assume uniformly distributed delays $\tau_{k}^{s c}$ and $\tau_{k}^{c a}$ as described earlier. Set the sampling time $h=0.1$. We take $\tau_{\max }^{s c}=0.02$ and $\tau_{\max }^{c a}=0.01$.

The computed sampled data system data are then

$$
\Phi=\left[\begin{array}{cc}
1.02122 & -0.003027 \\
0 & 0.99700
\end{array}\right], \Gamma_{0, a}=\left[\begin{array}{c}
0.08577 \\
0
\end{array}\right]
$$

Finally the covariance matrix $H$ is given by

$$
H=\left[\begin{array}{cc}
4.3180 \times 10^{-5} & 0 \\
0 & 0
\end{array}\right]
$$

From these data it is straightforward to write up the equivalent discrete time control problem as described earlier (can be done automatically within MATLAB) and to apply the DAM1 and DAM2 algorithms to compute the optimal feedback gain matrix $F_{o p}$.

The algorithms were initialised with $F_{0}=-0.4$ and $\epsilon=10^{-5}$. The DAM1 algorithm gave the result;

$$
F_{o p}=-9.9841
$$

with the associated optimal loss function value $J\left(F_{o p}\right)=0.11720$, in six iteration steps with one loss function evaluation per iteration step. The DAM2 algorithm gave the result $F_{o p}=-9.9839$, and the same loss value $J\left(F_{o p}\right)=0.11720$, from the same initialisation data in six iteration steps with one loss evaluation per iteration step. So although the DAM1 and DAM2 are not equivalent in this example, they both performed efficiently.

It should be emphasised that it is easy to compute not only static but also dynamic controllers even in the random delay case. To illustrate this important point let us consider the first order dynamic controller

$$
u(k h)=f_{1} y(t)+f_{2} u(t-1)
$$

By defining the augmented state vector $x_{a}(k h)=$ $\left[x(k h)^{\prime} u((k-1) h)\right]^{\prime}$ (see also eqn. 10) and the augmented output vector $y_{a}(k h)=[y(k h) u((k-1) h)]^{\prime}$, we can clearly write

$$
y_{a}(k h)=\left[\begin{array}{ll}
C & 0 \\
0 & 1
\end{array}\right] x_{a}(k h)+\left[\begin{array}{c}
e(k h) \\
0
\end{array}\right]
$$

Hence $u(k h)=\left[f_{1} f_{2}\right] y_{a}(k h)$ and so we are back in the standard problem formulation of Section 3. Thus we can find the optimal values of $f_{1}$ and $f_{2}$ using the DAM1 algorithm, say. This gives, with the initial values $f_{1}=-0.4$ 
and $f_{2}=0$, and otherwise with the same initialisation data as earlier, the optimal gain values

$$
F_{o p}=\left[\begin{array}{ll}
f_{1} & f_{2}
\end{array}\right]_{o p}=\left[\begin{array}{ll}
-10.001 & 0.0330
\end{array}\right]
$$

and the optimal loss value $J\left(F_{\text {op }}\right)=0.11701$ in nine iteration steps with one loss evaluation per iteration step.

We can clearly keep augmenting $x_{a}(k h)$ and $y_{a}(k h)$ with $y(k h), y((k-1) h), \ldots, u((k-1) h, \quad u((k-2) h), \ldots, \quad$ and still get from eqn. 10 an augmented problem of the standard form treated in Section 3. Hence dynamic controllers of any order can be optimised with the DAM1 and DAM2 algorithms also for the random delay case.

\section{Conclusions}

We have described a parametric LQ control framework for linear systems with random parameters. Specifically, we have derived the parametric LQ design equations for digital control of continuous-time systems with randomly varying delays between the sensor, controller and actuator nodes. This problem motivates in a nice manner the more general setup studied here. We have been especially interested to illustrate a very simple, yet efficient, approach for the numerical solution of such nonstandard LQ problems. By a suitable choice of augmented state systems, it is possible to consider both static and dynamic controllers within a single numerical framework. Robustness issues against, for example, modelling errors, unmodelled dynamics, varying disturbances and system delays can be addressed within this flexible framework.

\section{Acknowledgment}

Financial support to the first author from the Academy of Finland under grant 40536 is gratefully acknowledged.

\section{References}

1 ÅSTRÖM, K.J.: 'Introduction to stochastic control theory' (Academic Press, New York, 1970)

2 ANDERSON, B.D.O, and MOORE, J.B.: 'Linear optimal control' (Prentice-Hall, Englewood Cliffs, 1971)
3 DORATO, P., ABDALLAH, C., and CERONE, V.: 'Linear-quadratic control' (Prentice-Hall, Englewood Cliffs, 1995)

4 MÄKILÄ, P.M., and TOIVONEN, H.T.: 'Computational methods for parametric LQ problems - A survey', IEEE Trans. Autom. Control, 1987, AC-32, pp. $658-671$

5 BERNSTEIN, D.S., and HADDAD, W.M.: 'Optimal projection equations for discrete-time fixed-order dynamic compensation of linear systems with multiplicative white noise', Int. J. Control, 1987, 46, pp. $65-73$

6 LIU, K., SKELTON, R.E., and GRIGORIADIS, K.: 'Optimal controllers for finite wordlength implementation', IEEE Trans. Autom. Control, 1992, 37, pp. 1294-1304

7 HYLAND, D.C., and BERNSTEIN, D.S.: 'The optimal projection equations for model reduction and the relationships among the methods of Wilson, Skelton, and Moore', IEEE Trans. Autom. Control, 1985 , AC-30, pp. 1201-1211

8 MÄKILÄ, P.M.: 'Linear quadratic control revisited', Automatica, 2000 , 36. pp. 83-89

9 MÄKILÄ, P.M., PARTINGTON, J.R., and NORLANDER, T.: 'Bounded power signal spaces for robust control and modelling', SIAM J. Control Optim., 1998, 37, pp. 92-117

10 RAY, A.: 'Output feedback control under randomly varying distributed delays', J Guid. Control Dynam., 1994, 17, pp. 701-711

11 CHAN, H., and ÖZGÜNER, U.: 'Closed-loop control of systems over a communications network with queues', Int. J. Control, 1995, 62, pp. 493-510

12 RAY, A.: 'Distributed data communication networks for real-time process control', Chem. Eng. Commun., 1988, 65, pp. 139-154

13 OVASKA, S.I., and VAINIO, O.: 'Predictive compensation of timevarying computing delay on real-time control systems', IEEE Trans. Control Syst. Technol., 1997, 5, pp. 523-526

14 BELLE ISLE A.P, and KOZIN, F. 'On the almost-sure sample stability of systems with randomly time-varying delays', Automatica, 1972,8 , pp. $775-763$

15 WITTENMARK, B., NILSSON, J., and TÖRNGREN, M.: 'Timing problems in real-time control systems.'. Proc. American Control Conference, 1995, pp. 2000-2004

16 NILSSON, J.: "Real-time control systems with delays.". 1998, PhD Dissertation, Lund Institute of Technology, Department of Automatic Control

17 CHAN, H.: "Stability and control of systems over a communication network.". PhD Dissertation, Ohio State University, Department of network.". PhD Dissertation,

18 MÄKIL $\ddot{A}$, P.M.: 'Multiple models, multiplicative noise and linear quadratic control - algorithmic aspects', Int. J. Control, 1991, 54, pp. $921-941$

19 LANCASTER, P.: 'Explicit solutions of linear matrix equations', SIAM Rev. 1970,12 , pp. 544-566

20 HALYO, N and BROUSSARD, J.R. "A convergent algorithm for the stochastic infinite-time discrete optimal output feedback problem". Proc. Joint automatic control conference, 1981, Charlottesville

21 MÄKILÄ, P.M.: 'On the Anderson-Moore method for solving the optimal output feedback problem', IEEE Trans. Autom. Control, 1984, AC-29, pp. 834-836

22 MÄKILÄ, P.M., WESTERLUND, T., and TOIVONEN, H.T. 'Constrained linear quadratic Gaussian control with process applications', Automatica, 1984, 20, pp. 15-29

23 WESTERLUND, T: 'A digital quality control system for an industrial dry process cement kiln', IEEE Trans. Autom. Control, 1981, AC-26, pp. $885-890$ 